\title{
Spinning bodies in curved spacetime
}

\author{
G. d'Ambrosi, ${ }^{1, *}$ S. Satish Kumar, ${ }^{2, \dagger}$ J. van de Vis, ${ }^{1,2,}$ and J. W. van Holten ${ }^{1,2, \S}$ \\ ${ }^{1}$ Nikhef, Science Park 105, Amsterdam 1098XG, Netherlands \\ ${ }^{2}$ Lorentz Institute, Leiden University, Niels Bohrweg 2, Leiden 2333CA, Netherlands
}

(Received 26 November 2015; published 17 February 2016)

\begin{abstract}
We study the motion of neutral and charged spinning bodies in curved spacetime in the test-particle limit. We construct equations of motion using a closed covariant Poisson-Dirac bracket formulation that allows for different choices of the Hamiltonian. We derive conditions for the existence of constants of motion and apply the formalism to the case of spherically symmetric spacetimes. We show that the periastron of a spinning body in a stable orbit in a Schwarzschild or Reissner-Nordstrøm background not only precesses but also varies radially. By analyzing the stability conditions for circular motion we find the innermost stable circular orbit (ISCO) as a function of spin. It turns out that there is an absolute lower limit on the ISCOs for increasing prograde spin. Finally we establish that the equations of motion can also be derived from the Einstein equations using an appropriate energy-momentum tensor for spinning particles.
\end{abstract}

DOI: 10.1103/PhysRevD.93.044051

\section{INTRODUCTION}

Relativistic dynamics is becoming increasingly relevant in astrophysics and cosmology, for example when it comes to understanding compact stars, black holes, and gravitational waves. It was realized long ago that spin, i.e. the internal angular momentum of compact objects, can have important dynamical effects, and an extensive literature on the subject has become available [1-10].

In a recent article [11] three of us presented a nonstandard covariant description of spinning bodies in the testparticle limit. In contrast to the most-widely used approach of test-particle dynamics [12-16], it is formulated in terms of a covariant kinetic momentum rather than a canonical momentum which is not proportional to the four-velocity of the body. The price to pay is a world line that does not always coincide with that of a center of mass; rather it follows the spin, with the result that there is a mass dipole describing the displacement between the two in the presence of curvature. There are several advantages to this formulation: it does not require constraints like the Pirani or Tulczyjew conditions, it can be used with a variety of Hamiltonians, and it makes the analysis of the motion of spinning test particles in curved spacetime more tractable; in some cases of practical interest exact results are obtained.

In this paper we continue to develop this formalism of spin dynamics in the test-particle limit. It is organized as follows. In Sec. II we provide a brief summary of the Hamiltonian phase-space approach of Ref. [11], extending it to include also electric charge and electromagnetic fields. We proceed with the role of Killing vectors in constructing

\footnotetext{
"gdambros@nikhef.nl

satish@lorentz.leidenuniv.nl

jorindev@nikhef.nl

§.holten@ nikhef.nl
}

constants of motion in Sec. III. In Sec. IV the formalism is applied to motion associated with a minimal kinetic Hamiltonian in static and spherically symmetric spacetimes of Schwarzschild and Reissner-Nordstrøm type. We analyze circular orbits in Sec. V and find noncircular orbits by a perturbative construction in Sec. VI. We also determine the effects of spin on the periastron and show that in addition to precessing the periastron also shifts radially. In Sec. VII the stability of circular orbits is analyzed in detail to determine the radius of the innermost stable circular orbit (ISCO) as a function of spin. The stability conditions are found to impose an upper limit on the spin associated with the ISCO. Section VIII describes how to include Stern-Gerlach forces [17-21], both of electromagnetic and of gravitational origin, using a class of nonminimal Hamiltonians. We show that they allow for an extension of all the constants of motion associated with the minimal Hamiltonian and determine also the circular orbits for this case. In Sec. IX it is shown that the equations of motion we use can be derived in a different way from consistency of the Einstein equations with an appropriate energy-momentum tensor. Finally in Sec. $\mathrm{X}$ we conclude with a discussion and summary. Some mathematical details have been collected in the appendixes.

\section{COVARIANT HAMILTON FORMALISM FOR SPINNING BODIES}

Test particles are gravitational objects characterized by a finite number of degrees of freedom such as mass, charge, and spin, of which the backreaction on spacetime geometry can be considered negligible. Thus the phase space is finite dimensional; it is spanned by the position $x^{\mu}$, momentum $\pi_{\mu}$, and antisymmetric spin tensor $\Sigma^{\mu \nu}$, with mass $m$ and charge $q$ constant parameters characterizing the strength of interactions. While the four-velocity $u^{\mu}=\dot{x}^{\mu}$ is a timelike 
unit vector, the spin tensor can actually be decomposed in a spacelike vector $Z^{\mu}$, the mass dipole vector, and a spacelike axial vector $S_{\mu}$, the spin proper,

$$
\Sigma^{\mu \nu}=-\frac{1}{\sqrt{-g}} \varepsilon^{\mu \nu \kappa \lambda} u_{\kappa} S_{\lambda}+u^{\mu} Z^{\nu}-u^{\nu} Z^{\mu},
$$

where

$$
S_{\mu}=\frac{1}{2} \sqrt{-g} \varepsilon_{\mu \nu \kappa \lambda} u^{\nu} \Sigma^{\kappa \lambda}, \quad Z^{\mu}=\Sigma^{\mu \nu} u_{\nu}
$$

such that $S \cdot u=Z \cdot u=0$. It is interesting to note that in addition one can define a third spacelike vector

$$
W^{\mu}=-\frac{1}{\sqrt{-g}} \varepsilon^{\mu \nu \kappa \lambda} u_{\nu} S_{\kappa} Z_{\lambda}=\left(\Sigma^{\mu \nu}-u^{\mu} Z^{\nu}\right) Z_{\nu},
$$

orthogonal to the other ones,

$$
W \cdot u=W \cdot S=W \cdot Z=0 .
$$

Together $(u, S, Z, W)$ form a set of independent vectors, one timelike and three spacelike, which can be used to define a frame of basis vectors carried along the particle world line.

We build our formulation of the dynamics on a set of covariant Poisson-Dirac brackets that are closed in the sense of Jacobi identities and independent of the specific Hamiltonian,

$$
\begin{aligned}
\left\{x^{\mu}, \pi_{\nu}\right\} & =\delta_{\nu}^{\mu}, \\
\left\{\pi_{\mu}, \pi_{\nu}\right\} & =\frac{1}{2} \Sigma^{\kappa \lambda} R_{\kappa \lambda \mu \nu}+q F_{\mu \nu}, \\
\left\{\Sigma^{\mu \nu}, \pi_{\lambda}\right\} & =\Gamma_{\lambda \kappa}{ }^{\mu} \Sigma^{\nu \kappa}-\Gamma_{\lambda \kappa}{ }^{\nu} \Sigma^{\mu \kappa}, \\
\left\{\Sigma^{\mu \nu}, \Sigma^{\kappa \lambda}\right\} & =g^{\mu \kappa} \Sigma^{\nu \lambda}-g^{\mu \lambda} \Sigma^{\nu \kappa}-g^{\nu \kappa} \Sigma^{\mu \lambda}+g^{\nu \lambda} \Sigma^{\mu \kappa},
\end{aligned}
$$

with all other brackets vanishing. The structure functions appearing in these brackets are the metric, connection, and curvature tensor of the spacetime manifold, with the electromagnetic field strength $F_{\mu \nu}$ appearing as the structure function for the central charge $q$.

Next we have to specify a Hamiltonian to generate the equations of motion via the brackets (5). The minimal choice is the kinetic Hamiltonian

$$
H_{0}=\frac{1}{2 m} g^{\mu \nu}(x) \pi_{\mu} \pi_{\nu} .
$$

Other choices are possible, and we will discuss a specific example in Sec. VIII. The equations of motion derived from the Hamiltonian $H_{0}$ are

$$
\pi_{\mu}=g_{\mu \nu} m u^{\nu}, \quad m D_{\tau} u^{\mu}=\frac{1}{2} \Sigma^{\kappa \lambda} R_{\kappa \lambda \nu}{ }^{\mu} u^{\nu}+q F_{\nu}^{\mu} u^{\nu},
$$

for the world line, and

$$
D_{\tau} \Sigma^{\mu \nu}=0
$$

for the spin tensor; here $D_{\tau}$ is the pullback of the covariant derivative on the world line. It follows that the world line is a curve on which the spin tensor is covariantly constant. This does not hold for the vectors $S$ and $Z$ individually as they satisfy

$$
\begin{aligned}
& m D_{\tau} S_{\mu}=\frac{1}{2} \sqrt{-g} \varepsilon_{\mu \nu \kappa \lambda} \Sigma^{\kappa \lambda}\left(\frac{1}{2} \Sigma^{\alpha \beta} R_{\alpha \beta} \stackrel{\nu}{\sigma}+q F_{\sigma}^{\nu}\right) u^{\sigma}, \\
& m D_{\tau} Z^{\mu}=\Sigma^{\mu \nu}\left(\frac{1}{2} \Sigma^{\kappa \lambda} R_{\kappa \lambda \nu \sigma}+q F_{\nu \sigma}\right) u^{\sigma} .
\end{aligned}
$$

Comparing with the conventional analysis of spinning particle dynamics $[4,5,7]$ it is seen that the constraint on the mass dipole (e.g., the Pirani condition [22]) has been replaced here by a proper equation of motion. As a result in our formulation of classical relativistic mechanics the massdipole $Z$ cannot vanish permanently, except in flat field-free Minkowski spacetime. ${ }^{1}$ However, it is easily established that the two approaches can be made to agree to linear order in the spin tensor.

\section{CONSTANTS OF MOTION}

In the Hamiltonian formalism constants of motion are found by requiring its bracket with the Hamiltonian to vanish. There are three generic constants of motion for any spacetime geometry. First the Hamiltonian itself, which defines the particle mass

$$
H_{0}=-\frac{m}{2} \Rightarrow g_{\mu \nu} u^{\mu} u^{\nu}=-1
$$

In addition, there are two constants of motion for the spin: the total spin

$$
I=\frac{1}{2} g_{\mu \kappa} g_{\nu \lambda} \Sigma^{\mu \nu} \Sigma^{\kappa \lambda}=S \cdot S+Z \cdot Z
$$

and the pseudoscalar product

$$
D=\frac{1}{8} \sqrt{-g} \varepsilon_{\mu \nu \kappa \lambda} \Sigma^{\mu \nu} \Sigma^{\kappa \lambda}=S \cdot Z .
$$

\footnotetext{
${ }^{1}$ However, one can have $Z^{\mu}=0$ in pseudoclassical models in which the spin tensor is represented in terms of Grassmann variables $\Sigma^{\mu \nu}=i \psi^{\mu} \psi^{\nu}$, because of conservation of the supercharge $Q=\psi^{\mu} \pi_{\mu}[23-25]$.
} 
Furthermore there are constants of motion depending on the symmetries of the geometry. They are constructed in terms of Killing vectors and tensors. In particular, constants of motion $J$ of the form

$$
J=\gamma+\alpha^{\mu} \pi_{\mu}+\frac{1}{2} \beta_{\mu \nu} \Sigma^{\mu \nu}
$$

exist if

$$
\begin{aligned}
\nabla_{\mu} \alpha_{\nu}+\nabla_{\nu} \alpha_{\mu} & =0, \quad \nabla_{\lambda} \beta_{\mu \nu}=R_{\mu \nu \lambda \kappa} \alpha^{\kappa}, \\
\partial_{\mu} \gamma & =q F_{\mu \nu} \alpha^{\nu} .
\end{aligned}
$$

Thus $\alpha^{\mu}$ is a Killing vector and $\beta_{\mu \nu}$ its curl,

$$
\beta_{\mu \nu}=\frac{1}{2}\left(\nabla_{\mu} \alpha_{\nu}-\nabla_{\nu} \alpha_{\mu}\right),
$$

while a solution for $\gamma$ can be found if the Lie derivative of the vector potential with respect to $\alpha$ vanishes,

$$
\alpha^{\nu} \partial_{\nu} A_{\mu}+\partial_{\mu} \alpha^{\nu} A_{\nu}=0 \Rightarrow \gamma=q A_{\mu} \alpha^{\mu} .
$$

This requirement, in fact, states that the electromagnetic and gravitational fields must both exhibit the same symmetries for an associated constant of motion to exist.

\section{SPHERICAL SYMMETRY}

There are few static solutions of the Einstein equations possessing spherical symmetry. The most relevant ones are the Minkowski and Schwarzschild geometries. In addition, the Reissner-Nordstrøm geometry is a static and spherically symmetric solution of the Einstein-Maxwell equations. In these symmetric spacetimes the orbital angular momentum combines with the spin to create a conserved total angular momentum. In this section we consider the exterior Reissner-Nordstrøm geometry of an electrically charged massive spherical body and its reduction to Schwarzschild spacetime in the limit of vanishing charge.

The standard form of the Schwarzschild-ReissnerNordstrøm metric is represented by the line element

$$
\begin{aligned}
g_{\mu \nu} d x^{\mu} d x^{\nu}= & -\left(1-\frac{2 M}{r}+\frac{Q^{2}}{r^{2}}\right) d t^{2}+\frac{d r^{2}}{1-\frac{2 M}{r}+\frac{Q^{2}}{r^{2}}} \\
& +r^{2} d \theta^{2}+r^{2} \sin ^{2} \theta d \varphi^{2} .
\end{aligned}
$$

Here $M$ is the mass and $Q$ is the charge of the source creating the spacetime curvature as well as a static electric Coulomb field

$A=A_{\mu} d x^{\mu}=-\frac{Q}{r} d t, \quad \frac{1}{2} F=d A=\frac{Q}{r^{2}} d r \wedge d t$.
The components of the connection and curvature tensor are collected in Appendix A. In the line element (17) $t$ and $\varphi$ are cyclic coordinates implying simple constant Killing vectors for time translations and rotations around the $z$ axis. Two more Killing vectors exist generating rotations around the two other axes. As the Coulomb potential is also static and spherically symmetric, we obtain four constants of motion: (i) the particle energy

$$
E=-\pi_{t}+\frac{q Q}{r}-\left(\frac{M}{r^{2}}-\frac{Q^{2}}{r^{3}}\right) \Sigma^{t r},
$$

with $Q=0$ in Schwarzschild spacetime; (ii) and the threevector of total angular momentum

$$
\begin{aligned}
J_{1}= & -\sin \varphi \pi_{\theta}-\operatorname{cotan} \theta \cos \varphi \pi_{\varphi}-r \sin \varphi \Sigma^{r \theta} \\
& -r \sin \theta \cos \theta \cos \varphi \Sigma^{r \varphi}+r^{2} \sin ^{2} \theta \cos \varphi \Sigma^{\theta \varphi}, \\
J_{2}= & \cos \varphi \pi_{\theta}-\operatorname{cotan} \theta \sin \varphi \pi_{\varphi}+r \cos \varphi \Sigma^{r \theta} \\
& -r \sin \theta \cos \theta \sin \varphi \Sigma^{r \varphi}+r^{2} \sin ^{2} \theta \sin \varphi \Sigma^{\theta \varphi}, \\
J_{3}= & \pi_{\varphi}+r \sin ^{2} \theta \Sigma^{r \varphi}+r^{2} \sin \theta \cos \theta \Sigma^{\theta \varphi} .
\end{aligned}
$$

The analysis of the equations of motion can be simplified considerably by using the spherical symmetry to fix the direction of the total angular momentum to be the $z$ axis,

$$
\mathbf{J}=(0,0, J), \quad J=m r^{2} u^{\varphi}+r \Sigma^{r \varphi},
$$

resulting in

$$
\Sigma^{r \theta}=-m r u^{\theta}, \quad \Sigma^{\theta \varphi}=\frac{J}{r^{2}} \operatorname{cotan} \theta .
$$

Clearly this choice does not fix the motion to be in the equatorial plane $\theta=\pi / 2$, as the spin and orbital angular momentum are not necessarily aligned; indeed, it is possible to have precession of both the spin and the orbital angular momentum, making the plane of the orbit precess [26] as well.

\section{CIRCULAR ORBITS}

In a Schwarzschild-Reissner-Nordstrøm background planar motion requires alignment of the spin and orbital angular momentum. In this section we consider, in particular, the conditions for plane circular motion. The analysis is most conveniently done by taking the plane of motion to be the equatorial plane, which implies $\theta=$ $\pi / 2$ and $u^{\theta}=0$ as well as $\dot{u}^{\theta}=0$. As a result

$$
\Sigma^{t \theta}=\Sigma^{r \theta}=\Sigma^{\theta \varphi}=0 .
$$

From the definition (12) it follows that such orbits satisfy the conservation law 


$$
D=S \cdot Z=0 \text {. }
$$

For circular motion we additionally impose $r=R=$ const and $u^{r}=\dot{u}^{r}=0$.

Using the equation of motion (7) for the $r$-coordinate, the latter condition leads to

$$
\begin{aligned}
( & \left.M R^{3}-3 M^{2} R^{2}-3 R^{2} Q^{2}+6 M R Q^{2}-2 Q^{4}\right)\left(R^{2}-2 M R+Q^{2}\right) u^{t 2} \\
& -\left(R^{2}-3 M R+2 Q^{2}\right)\left(M R-Q^{2}\right) R^{4} u^{\varphi 2} \\
= & {\left[\left(2 M R-3 Q^{2}\right) \varepsilon-\left(M R-2 Q^{2}\right) \frac{q Q}{m R}\right]\left(R^{2}-2 M R+Q^{2}\right) R^{2} u^{t}+\left(M R-Q^{2}\right)^{2} \eta R^{2} u^{\varphi}, }
\end{aligned}
$$

where $\varepsilon=E / m$ and $\eta=J / m$ are the particle energy and angular momentum per unit of mass. The Hamiltonian constraint (10) for circular orbits becomes

$$
\left(1-\frac{2 M}{R}+\frac{Q^{2}}{R^{2}}\right) u^{t 2}=1+R^{2} u^{\varphi 2}
$$

Hence (25) and (26) constitute two independent equations for $u^{t}$ and $u^{\varphi}$ in terms of $(M, Q, R)$ and $(\varepsilon, \eta)$; therefore $u^{t}$ and $u^{\varphi}$ are constant on circular orbits,

$$
\dot{u}^{t}=\dot{u}^{\varphi}=0
$$

As a result, on circular orbits the equations of motion for $u^{t}$ and $u^{\varphi}$ reduce to

$$
m g_{t t} \dot{u}^{t}=\Sigma^{t \varphi} R_{t \varphi t \varphi} u^{\varphi}=0, \quad m g_{\varphi \varphi} \dot{u}^{\varphi}=\Sigma^{t \varphi} R_{t \varphi t \varphi} u^{t}=0,
$$

implying that $\Sigma^{t \varphi}=0$. This in turn also requires $\dot{\Sigma}^{t \varphi}=0$, or

$$
\left(M R-Q^{2}\right) u^{t} \Sigma^{r \varphi}+\left(R^{2}-2 M R+Q^{2}\right) u^{\varphi} \Sigma^{t r}=0 .
$$

Using the conservation laws for $E$ and $J$, this can be replaced by

$$
\begin{gathered}
\left(M R-Q^{2}\right)^{2} \eta u^{t}-\left(R^{2}-2 M R+Q^{2}\right)\left(\varepsilon-\frac{q Q}{m R}\right) R^{4} u^{\varphi} \\
+\left(R^{2}-3 M R+2 Q^{2}\right)(R-M) R^{3} u^{t} u^{\varphi}=0 .
\end{gathered}
$$

As this is yet another equation between $\left(u^{t}, u^{\varphi}\right)$ and the constants of motion, it fixes a relation between the energy $E=m \varepsilon$ and total angular momentum $J=m \eta$ for a given circle $r=R$. In fact, we can now eliminate $\eta$ between Eqs. (25) and (30), using the constraint (26), to get

$$
\begin{aligned}
& \left(R^{3}-3 M R^{2}+3 M^{2} R-M Q^{2}\right) u^{t 3}-\left(R^{2}-3 M R+2 Q^{2}\right) R u^{t} \\
& \quad=-R^{3}\left(\varepsilon-\frac{q Q}{m R}\right)+\left[\left(R^{2}-2 Q^{2}\right) \varepsilon-\left(R^{2}-M R-Q^{2}\right) \frac{q Q}{m R}\right] R u^{t 2} .
\end{aligned}
$$

For the case of Schwarzschild geometry we take $Q=0$ to get

$$
\varepsilon R^{2}\left(1-u^{t 2}\right)=R(R-3 M) u^{t}-\left(R^{2}-3 M R+3 M^{2}\right) u^{t 3} .
$$

It is straightforward to check that in the spinless case with

$$
u^{t}=\frac{\varepsilon}{1-\frac{2 M}{R}},
$$

this relation reduces to the standard result

$$
\varepsilon=\frac{1-\frac{2 M}{R}}{\sqrt{1-\frac{3 M}{R}}}
$$

for circular orbits.

Having solved Eq. (31) —or (32) if appropriate—one can then also compute the orbital angular momentum per unit of mass from the Hamiltonian constraint,

$$
\ell^{2}=R^{4} u^{\varphi 2}=\left(R^{2}-2 M R+Q^{2}\right) u^{t 2}-R^{2} .
$$

For neutral particles $(q=0)$ instead of eliminating $\eta$ and $u^{\varphi}$ from Eqs. (25) and (30), one can alternatively eliminate $\varepsilon$ and $u^{t}$ to get a relation for the total angular momentum per unit of mass $\eta$ and the proper angular velocity $u^{\varphi}$, 


$$
\begin{aligned}
\eta( & \left.M R-Q^{2}\right)^{2}\left[2 M R-3 Q^{2}+R^{2} u^{\varphi 2}\left(R^{2}-2 Q^{2}\right)\right] \\
= & R^{2} u^{\varphi}\left(M^{2} R^{4}-2 M^{2} R^{2} Q^{2}-2 M R^{3} Q^{2}+R^{2} Q^{4}+4 M R Q^{4}-2 Q^{6}\right) \\
& -R^{4} u^{\varphi 3}\left(M R^{5}-6 M^{2} R^{4}+6 M^{3} R^{3}-R^{4} Q^{2}+10 M R^{3} Q^{2}-11 M^{2} R^{2} Q^{2}\right. \\
& \left.-4 R^{2} Q^{4}+5 M R Q^{4}\right) .
\end{aligned}
$$

In Schwarzschild spacetime this reduces to the result quoted in Ref. [11],

$$
\frac{\eta}{M}\left(2+\frac{R^{3} u^{\varphi 2}}{M}\right)=\frac{R^{3} u^{\varphi}}{M^{2}}\left[1-\frac{R^{3} u^{\varphi 2}}{M}\left(1-\frac{6 M}{R}+\frac{6 M^{2}}{R^{2}}\right)\right] .
$$

\section{PLANE NONCIRCULAR ORBITS}

In general relativity the standard procedure for comparing geodesics is the method of geodesic deviations. It is based on a covariant definition of differences between geometric quantities associated with geodesics, like the unit tangent vectors defining the proper four-velocities of test particles. The procedure can be generalized to world lines of particles carrying charge and/or spin as follows.

Consider two solutions $\left(x^{\mu}(\tau), u^{\mu}(\tau), \Sigma^{\mu \nu}(\tau)\right)$ and $\left(\bar{x}^{\mu}(\tau)\right.$, $\left.\bar{u}^{\mu}(\tau), \bar{\Sigma}^{\mu \nu}(\tau)\right)$ of the equations of motion (7) and (8). The direct differences between dynamical quantities on each world line at equal proper time $\tau$ are denoted by $\delta$,

$$
\delta X(\tau)=\bar{X}(\tau)-X(\tau)
$$

for any $X=\left(x^{\mu}, u^{\mu}, \Sigma^{\mu \nu}\right)$. As the coordinates $x^{\mu}$ are spacetime scalars, the velocities $u^{\mu}$ spacetime vectors, and the spin-dipoles $\Sigma^{\mu \nu}$ spacetime tensors, we can define their covariant differences by parallel displacement,

$$
\begin{aligned}
\Delta x^{\mu}(\tau) & =\delta x^{\mu}(\tau), \quad \Delta u^{\mu}=\delta u^{\mu}(\tau)+\delta x^{\lambda} \Gamma_{\lambda \nu}{ }^{\mu} u^{\nu}, \\
\Delta \Sigma^{\mu \nu} & =\delta \Sigma^{\mu \nu}+\delta x^{\lambda} \Gamma_{\lambda \kappa}{ }^{\mu} \Sigma^{\kappa \nu}+\delta x^{\lambda} \Gamma_{\lambda \kappa}{ }^{\nu} \Sigma^{\mu \kappa} .
\end{aligned}
$$

The equations of motion now imply equations for the proper-time dependence of these covariant variations; to linear order,

$$
\begin{aligned}
\Delta u^{\mu} & =D_{\tau} \Delta x^{\mu}, \\
D_{\tau}^{2} \Delta x^{\mu}-R_{\lambda \kappa}{ }^{\mu}{ }_{\nu} u^{\kappa} u^{\nu} \Delta x^{\lambda} & =\frac{1}{2 m} \Sigma^{\rho \sigma} R_{\rho \sigma}{ }_{\nu}{ }_{\nu} D_{\tau} \Delta x^{\nu}+\frac{1}{2 m} \Sigma^{\rho \sigma} \nabla_{\lambda} R_{\rho \sigma}{ }_{\nu}{ }_{\nu} u^{\nu} \Delta x^{\lambda} \\
& +\frac{1}{2 m} \Delta \Sigma^{\rho \sigma} R_{\rho \sigma}{ }^{\mu}{ }_{\nu} u^{\nu}+\frac{q}{m} F^{\mu}{ }_{\nu} D_{\tau} \Delta x^{\nu}+\frac{q}{m} \nabla_{\lambda} F^{\mu}{ }_{\nu} u^{\nu} \Delta x^{\lambda}, \\
D_{\tau} \Delta \Sigma^{\mu \nu}+\left(R_{\lambda \kappa \sigma}{ }^{\mu} \Sigma^{\sigma \nu}-R_{\lambda \kappa \sigma}{ }^{\nu} \Sigma^{\sigma \mu}\right) u^{\kappa} \Delta x^{\lambda} & =0 .
\end{aligned}
$$

The formalism, eventually with higher-order extensions [27-29], can be applied to a perturbative construction of world lines starting from a known solution of the equations of motion. The circular orbits found in the previous section define such a starting point to construct eccentric planar or nonplanar bound orbits in Schwarzschild-ReissnerNorstrøm backgrounds. However, computationally it is simpler to work with the noncovariant variations (38) rather than the covariant ones (39).

Considering eccentric orbits in the equatorial plane we keep the conditions (23) and (24). With these restrictions the allowed deviations from circular orbits can be parametrized by $\delta x^{\mu}=(\delta t, \delta r, \delta \varphi)$ for the orbital and $\delta \Sigma^{\mu \nu}=\left(\delta \Sigma^{t r}, \delta \Sigma^{r \varphi}, \delta \Sigma^{t \varphi}\right)$ for the spin degrees of freedom. Now using the conservation laws the variations $\delta \Sigma^{t r}$ and $\delta \Sigma^{r \varphi}$ can equivalently be expressed by the change in energy $\delta \varepsilon$ and total angular momentum $\delta \eta$ and the coordinate variations. In a condensed notation the relevant linearized deviation equations (40) then reduce to

$$
\left(\begin{array}{cccc}
\frac{d^{2}}{d \tau^{2}} & 0 & \alpha \frac{d}{d \tau} & \beta \\
0 & \frac{d^{2}}{d \tau^{2}} & \gamma \frac{d}{d \tau} & \zeta \\
\kappa \frac{d}{d \tau} & \lambda \frac{d}{d \tau} & \frac{d^{2}}{d \tau^{2}}+\mu & 0 \\
\nu \frac{d}{d \tau} & \sigma \frac{d}{d \tau} & \chi & \frac{d}{d \tau}
\end{array}\right)\left(\begin{array}{c}
\delta t \\
\delta \varphi \\
\delta r \\
\delta \Sigma^{t \varphi}
\end{array}\right)=\left(\begin{array}{c}
0 \\
0 \\
a \delta \eta+b \delta \varepsilon \\
c \delta \eta+d \delta \varepsilon
\end{array}\right),
$$

where the coefficients are defined in terms of the parameters of the circular reference orbit; the explicit expressions are given in Appendix B.

The general solution of the inhomogenous linear equations (41) can be decomposed in a specific solution plus a solution of the homogeneous equation. Now it is easy to 
find a simple specific solution: a constant shift $\delta r$ such that the new circular orbit has the same energy $\varepsilon^{\prime}=\varepsilon+\delta \varepsilon$ and total angular momentum $\eta^{\prime}=\eta+\delta \eta$ as the noncircular orbit we wish to construct. Hence by taking this special circular orbit as the reference orbit we fix $\delta \varepsilon=\delta \eta=0$, and we only have to solve the homogeneous equation (41).

To solve the homogeneous equation, we consider the characteristic equation for the periodic eigenfunctions of the operator (41),

$$
\omega^{3}\left(\omega^{4}-A \omega^{2}+B\right)=0,
$$

where

$$
\begin{aligned}
& A=\mu-\alpha \kappa-\beta \nu-\gamma \lambda-\zeta \sigma, \\
& B=\beta(\kappa \chi-\mu \nu+\gamma(\lambda \nu-\kappa \sigma))+\zeta(\lambda \chi-\mu \sigma-\alpha(\lambda \nu-\kappa \sigma)) .
\end{aligned}
$$

In addition to three 0-modes for potential secular solutions familiar from the motion of spinless particles [27,28], there are two pairs of nontrivial periodic solutions with angular frequencies

$$
\omega_{ \pm}^{2}=\frac{1}{2}\left(A \pm \sqrt{A^{2}-4 B}\right) .
$$

These periodic solutions can be cast in the simple form

$$
\begin{aligned}
\delta t & =n_{+}^{t} \sin \omega_{+}\left(\tau-\tau_{+}\right)+n_{-}^{t} \sin \omega_{-}\left(\tau-\tau_{-}\right), \\
\delta \varphi & =n_{+}^{\varphi} \sin \omega_{+}\left(\tau-\tau_{+}\right)+n_{-}^{\varphi} \sin \omega_{-}\left(\tau-\tau_{-}\right), \\
\delta r & =n_{+}^{r} \cos \omega_{+}\left(\tau-\tau_{+}\right)+n_{-}^{r} \cos \omega_{-}\left(\tau-\tau_{-}\right), \\
\delta \Sigma^{t \varphi} & =n_{+}^{\sigma} \sin \omega_{+}\left(\tau-\tau_{+}\right)+n_{-}^{\sigma} \sin \omega_{-}\left(\tau-\tau_{-}\right),
\end{aligned}
$$

where $\tau_{ \pm}$are constants of integration determining the relative phases of the oscillations, and up to some common normalization constants $C_{ \pm}$the amplitudes are

$$
\begin{aligned}
& n_{ \pm}^{t}=C_{ \pm}\left[\lambda(\beta \gamma-\alpha \zeta)+\beta\left(\omega_{ \pm}^{2}-\mu\right)\right], \\
& n_{ \pm}^{\varphi}=C_{ \pm}\left[-\kappa(\beta \gamma-\alpha \zeta)+\zeta\left(\omega_{ \pm}^{2}-\mu\right)\right], \\
& n_{ \pm}^{r}=C_{ \pm} \omega_{ \pm}(\beta \kappa+\zeta \lambda), \\
& n_{ \pm}^{\sigma}=C_{ \pm} \omega_{ \pm}^{2}\left(\omega_{ \pm}^{2}-\mu+\alpha \kappa+\gamma \lambda\right) .
\end{aligned}
$$

The null solutions of Eq. (42) suggest that in addition to the periodic solutions (45) there might also be secular solutions for the orbital degrees of freedom [27]. However, as we have chosen the energy and total angular momentum of the orbit to be the same as that of the circular reference orbit, no such freedom is left in this case, except for trivial shifts in the origin of the $t$ and $\varphi$ coordinates. Therefore the complete first-order solution for the noncircular planar orbits is

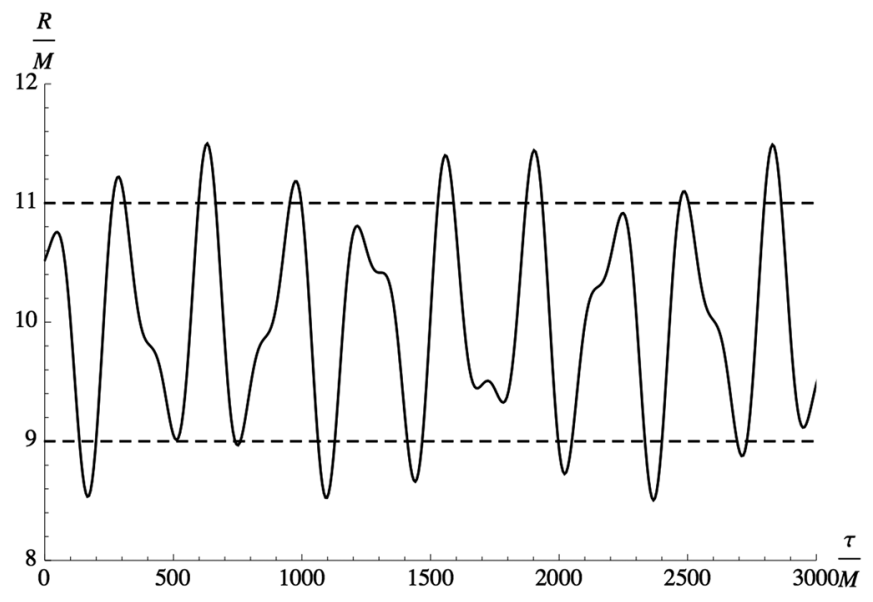

FIG. 1. Radial deviation from circular orbit with $R=10 M$ and $\ell=4 M$ as a function of proper time in Schwarzschild spacetime for deviation parameters as in Eq. (48).

$$
\begin{aligned}
& t(\tau)=u^{t} \tau+n_{+}^{t} \sin \omega_{+}\left(\tau-\tau_{+}\right)+n_{-}^{t} \sin \omega_{-}\left(\tau-\tau_{-}\right), \\
& \varphi(\tau)=u^{\varphi} \tau+n_{+}^{\varphi} \sin \omega_{+}\left(\tau-\tau_{+}\right)+n_{-}^{\varphi} \sin \omega_{-}\left(\tau-\tau_{-}\right), \\
& r(\tau)=R+n_{+}^{r} \cos \omega_{+}\left(\tau-\tau_{+}\right)+n_{-}^{r} \cos \omega_{-}\left(\tau-\tau_{-}\right), \\
& \Sigma^{t \varphi}(\tau)=n_{+}^{\sigma} \sin \omega_{+}\left(\tau-\tau_{+}\right)+n_{-}^{\sigma} \sin \omega_{-}\left(\tau-\tau_{-}\right) .
\end{aligned}
$$

The perturbations, in particular those in the radial direction, have double periods. Hence the periastron and apastron will behave in a complicated way, as the body reaches different minimal or maximal radial distances at nonconstant intervals. However, in the limit $B \ll A^{2}$ the dominant frequency will be $\omega_{+} \simeq \sqrt{A}$, and the variations in the periastron and apastron will be relatively slow. An example for the case of Schwarzschild geometry $(Q=0)$ is given in Fig. 1, where we have plotted the radial variation as a function of proper time for a circular reference orbit $R=10 M$ with orbital angular momentum $\ell=4 M$ and for deviation parameters

$$
n_{+}^{r}=0.1 R, \quad n_{-}^{r}=0.05 R, \quad \tau_{+}-\tau_{-}=100 M .
$$

As we have obtained the noncircular orbits (47) under the conditions that the specific energy and total angular momentum are the same as those of the circular reference orbits, the conservation laws (19) and (21) for the spindipole components link the variations $\delta \Sigma^{\mu \nu}$ of these quantities to those of the orbital parameters

$$
\delta \Sigma^{r \varphi}=F \delta u^{\varphi}+G \frac{\delta r}{R}, \quad \delta \Sigma^{t r}=K \delta u^{t}+L \frac{\delta r}{R},
$$

where 


$$
\begin{aligned}
F & =-m R, \quad G=-\frac{m}{R}\left(\eta+R^{2} u^{\varphi}\right), \\
K & =\frac{m R^{3}}{M R-Q^{2}}\left(1-\frac{2 M}{R}+\frac{Q^{2}}{R^{2}}\right), \\
L & =\frac{m R^{4}}{\left(M R-Q^{2}\right)^{2}}\left[\left(\frac{2 M}{R}\left(1-\frac{M}{R}+\frac{2 Q^{2}}{R^{2}}\right)-\frac{Q^{2}}{R^{2}}\left(3+\frac{Q^{2}}{R^{2}}\right)\right) u^{t}\right. \\
& \left.-\left(\frac{2 M}{R}-\frac{3 Q^{2}}{R^{2}}\right) \varepsilon+\frac{q Q}{m R}\left(\frac{M}{R}-\frac{2 Q^{2}}{R^{2}}\right)\right] .
\end{aligned}
$$

As a result

$$
\begin{aligned}
\delta \Sigma^{r \varphi} & =N_{+}^{r \varphi} \cos \omega_{+}\left(\tau-\tau_{+}\right)+N_{-}^{r \varphi} \cos \omega_{-}\left(\tau-\tau_{-}\right), \\
\delta \Sigma^{t r} & =N_{+}^{t r} \cos \omega_{+}\left(\tau-\tau_{+}\right)+N_{-}^{t r} \cos \omega_{-}\left(\tau-\tau_{-}\right),
\end{aligned}
$$

with

$$
N_{ \pm}^{r \varphi}=\omega_{ \pm} F n_{ \pm}^{\varphi}+G \frac{n_{ \pm}^{r}}{R}, \quad N_{ \pm}^{t r}=\omega_{ \pm} K n_{ \pm}^{t}+L \frac{n_{ \pm}^{r}}{R} .
$$

Thus we have obtained a large class of noncircular planar orbits (to first order in the deviations), parametrized by the constants $C_{ \pm}$and the radial coordinate $R$ of the circular orbit with the same specific energy and total angular momentum.

\section{THE ISCO}

In black-hole spacetimes there is an ISCO at a specific distance from the horizon. For simple point masses in Schwarzschild spacetime this orbit is located at $R=6 M$. Here the effective potential has a flex point where the orbital angular momentum reaches a minimum of $\ell^{2}=12 M^{2}$. Circular orbits at values $R<6 M$ are possible in principle but not in practice, as they correspond to maxima of the effective potential rather than minima; thus they are unstable under small perturbations.

The presence of spin alters the stability conditions and therefore the location of the ISCO. The stability conditions for circular orbits can be derived directly from the analysis in the previous section. Indeed, circular orbits are stable as long as the planar deviations display oscillatory behavior. In contrast, whenever the frequency $\omega$ of these deviations develops an imaginary part, the radial motion displays exponential behavior and the orbit becomes unstable [30,31]. Now the frequencies of the deviations are solutions of the eigenvalue equation (44). Thus we must ask what is the parameter domain in which the eigenvalues are real, and especially where the boundary between stability and instability is located. The first condition is obviously for $\omega_{ \pm}^{2}$ to be real; this requires

$$
A^{2}-4 B \geq 0 \text {. }
$$

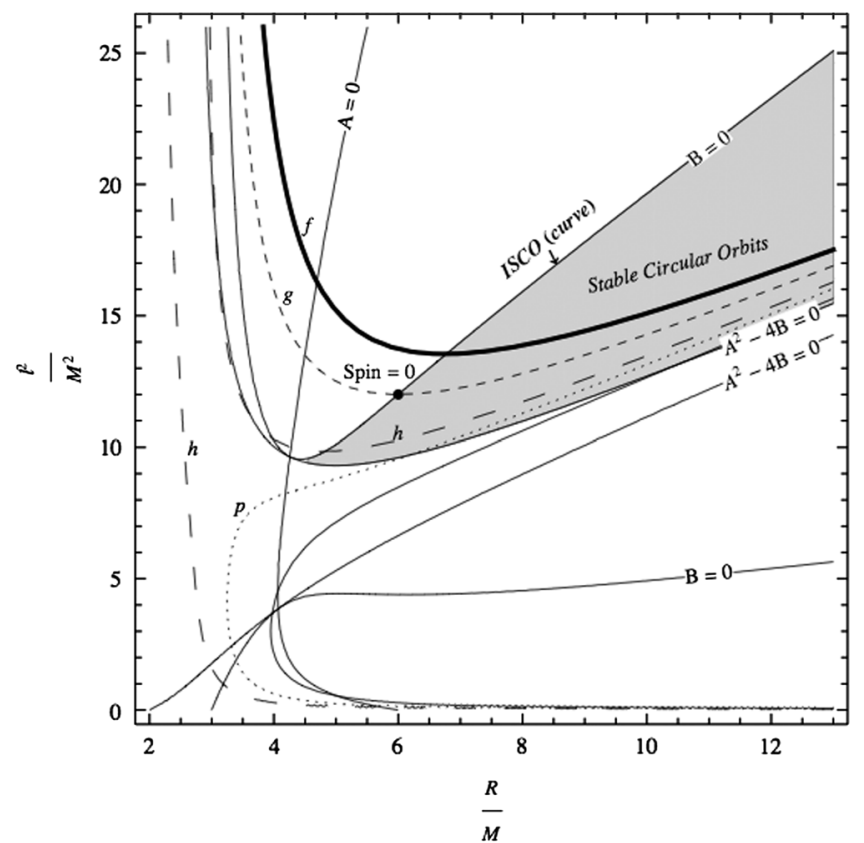

FIG. 2. Allowed domains of radius $R / M$ and orbital angular momentum $\ell / M$ for plane circular orbits in Schwarzschild spacetime. Included are four curves labeled $f, g, h, p$ defining orbits of fixed spin per unit of mass $\sigma$ for retrograde, vanishing, and prograde spin $\sigma / M=(-0.5,0,0.5,0.7)$.

In addition, for the frequencies $\omega_{ \pm}$themselves to be real as well we must demand that $\omega_{ \pm}^{2} \geq 0$, which happens if

$$
A \geq 0, \quad B \geq 0 .
$$

Figure 2 shows the solutions of these inequalities for the case of Schwarzschild spacetime in terms of the allowed values of the dimensionless radial coordinate $R / M$ and of the orbital angular momentum per unit of mass

$$
\ell=R^{2} u^{\varphi}
$$

The shaded area corresponds to stable circular orbits. As we have established in Sec. V that any circular orbit is determined for a given background geometry by the parameters $R$ and $J$ - which fix $u^{\varphi}, u^{t}$, and $E$ - the allowed orbits for fixed $R / M$ and various $\ell / M$ in Fig. 2 differ in the values of $\eta=J / m$. Equivalently they differ in the value of the spin per unit of mass parametrized by the dimensionless variable

$$
\frac{\sigma}{M}=\frac{R \Sigma^{r \varphi}}{m M}
$$

We have also indicated in Fig. 2 several curves of constant spin. The curves $g, f, h$ represent isospin lines for spin $\sigma=0$, for retrograde spin $\sigma=-0.5 M$, and for prograde spin $\sigma=0.5 \mathrm{M}$, respectively. In each case the ISCO is defined by the value of $R / M$ where the curve crosses the line $B=0$. For vanishing spin this is at the 


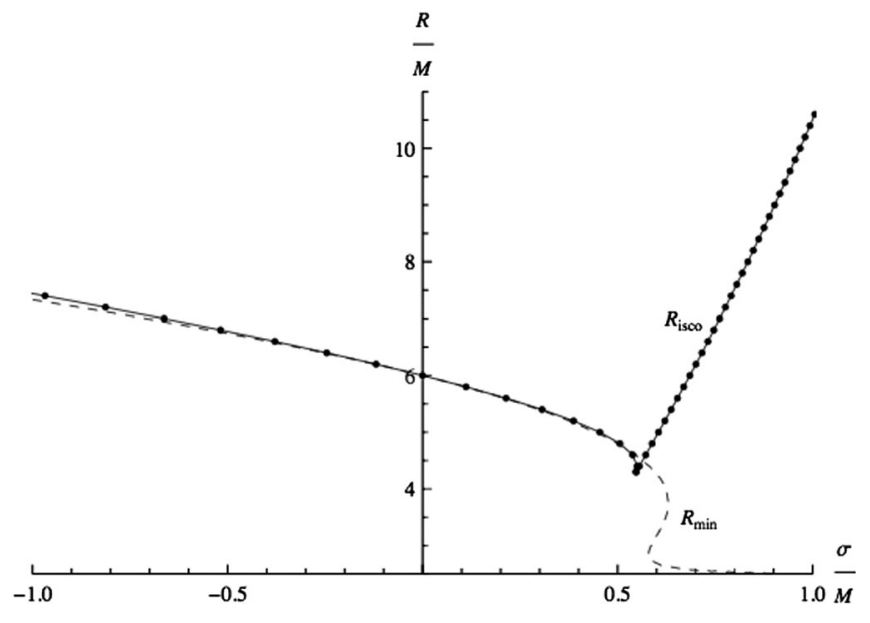

FIG. 3. Radius of the ISCO: $R_{\text {isco }} / M$, as a function of $\operatorname{spin} \sigma / M$ for Schwarzschild spacetime (continuous curve) compared with the radius of minimal orbital angular momentum $R_{\min } / M$ at fixed spin (dashed curve).

well-known value $R=6 M$, for prograde spin it is at a lower value of $R$, and for retrograde spin it is at a higher value of $R$. There actually is a smallest ISCO $R \simeq 4.5 M$ for $\sigma \simeq 0.55 M$, where the curve $B=0$ reaches a minimum value of orbital angular momentum.

For higher spin values the ISCO is reached at the point where the isospin curves cross the line $A^{2}-4 B=0$; an example is the curve labeled $p$ corresponding to $\sigma=0.7 \mathrm{M}$. In general such high values of $\sigma / M$ are possible only if the masses $m$ and $M$ of the test particle and the black hole creating the background become comparable. Of course, the backreaction of the test mass can then no longer be ignored, and our estimates of the ISCO become unreliable.

By calculating the values of the spin parameter $\sigma / M$ on the lines separating regions of stable and unstable orbits, we have extracted the values of $R$ for the ISCO as a function of $\sigma / M$ in Schwarzschild spacetime. The result is represented by the continuous curve labeled $R_{\text {isco }}$ in Fig. 3. The two branches correspond to lower-spin ISCOs on the curve $B=0$ and higher-spin ISCOs on the curve $A^{2}-4 B=0$, respectively. These results agree qualitatively with other studies in the literature based on the conventional Mathisson-Papapetrou-Dixon approach [30,32].

The isospin curves in Fig. 2 for lower-spin values, corresponding to the left-hand branch in Fig. 3, also suggest that the circular orbits become unstable when the orbital angular momentum reaches its minimum as a function of radial distance for constant $\sigma$. This issue can be analyzed more precisely by returning to Eq. (37) and rewriting it in the form

$$
\frac{\sigma R}{M^{2}}\left(\frac{2 R}{M}+\frac{\ell^{2}}{M^{2}}\right)=\frac{\ell}{M}\left(\frac{R}{M}-2\right)\left[\frac{R^{2}}{M^{2}}-\frac{\ell^{2}}{M^{2}}\left(\frac{R}{M}-3\right)\right] .
$$

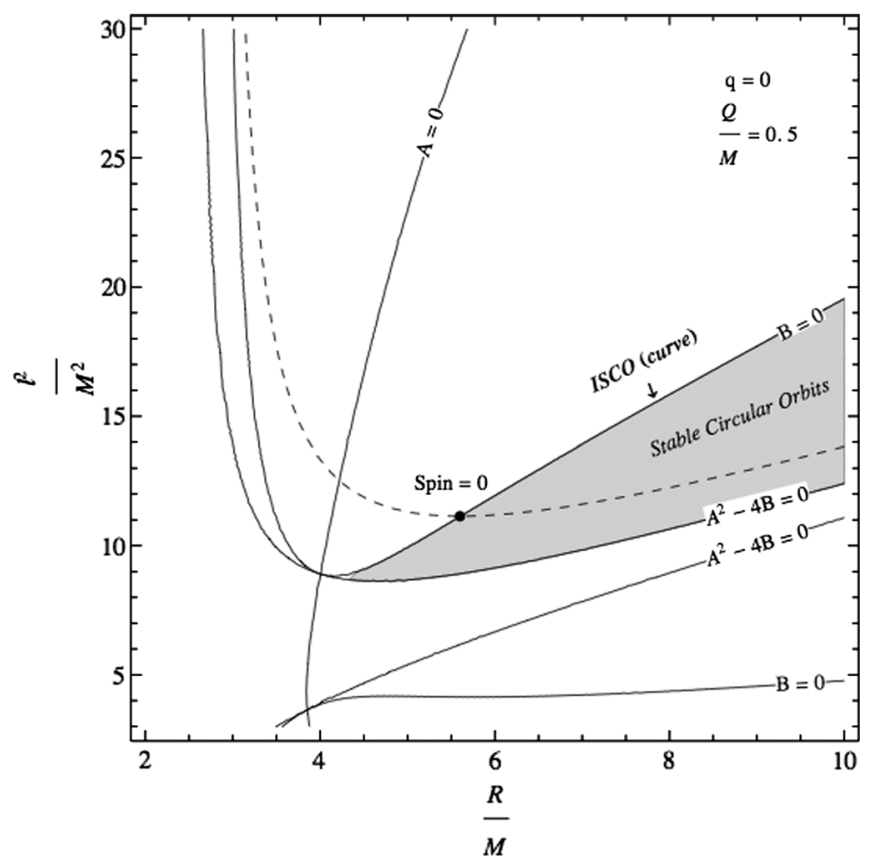

FIG. 4. Stable circular orbits of a chargeless spinning particle in Reissner-Nordstrøm spacetime with $Q / M=0.5$.

From this equation one can derive the minimum of $\ell / M$ as a function of $R / M$ for fixed spin $\sigma$. The result is plotted as the dashed curve labeled $R_{\min }$ in Fig. 3. Over the range of predominant physical interest $-0.5<\sigma / M<0.5$ the curve nearly coincides with that for $R_{\mathrm{isco}}$. However, there are small differences for larger absolute spin values, reminiscent of those found in higher-order post-newtonian corrections for compact binaries [31]. Note that the parts of the dashed curve for large retrograde spin actually enter the region of instability and hence do not correspond to stable circular orbits.

A similar analysis can be done for the case of a spinning test particle in a Reissner-Nordstrøm background. Of course, one has two more parameters to account for: the charge $Q$ of the central black hole, and the charge $q$ of the

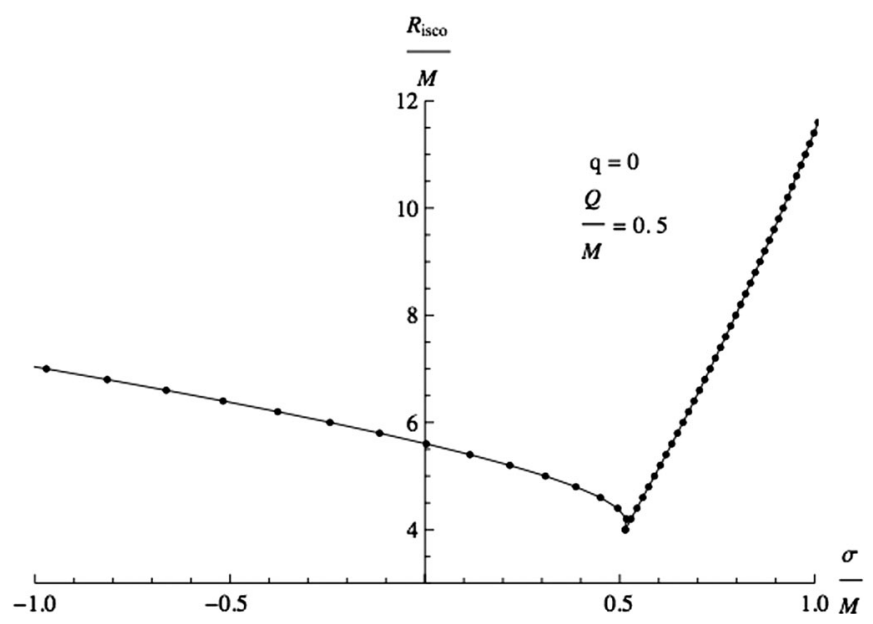

FIG. 5. $\quad R_{\text {isco }} / M$ as a function of $\sigma / M$ for Reissner-Norstrøm spacetime with $Q / M=0.5$. 
test particle. Figure 4 shows the stability region of circular orbits of electrically neutral spinning test particles for a black hole of charge $Q=0.5 M$, including the curve representing the $l-R$ relation for circular orbits of a spin-0 particle. In Fig. 5 the radial coordinate of the ISCO is given as a function of spin for the same black-hole background geometry with $Q=0.5 M$. We observe that the ISCOs are located closer to the horizon, $R_{\text {hor }} \simeq 1.87 M$, and that with this value of the black-hole charge the minimal ISCO is reached at a somewhat smaller spin value than in the Schwarzschild case, $\sigma_{\min } \simeq 0.53 M$.

\section{A NONMINIMAL HAMILTONIAN}

In the presence of external fields spinning particles can be subject to spin-dependent forces coupling to gradients in the fields like the well-known Stern-Gerlach force [21,33-35] in electrodynamics. Such forces can be modeled in our approach by additional spin-dependent terms in the Hamiltonian,

$$
H=H_{0}+H_{\mathrm{SG}}, \quad H_{\mathrm{SG}}=\frac{\kappa}{4} R_{\mu \nu \kappa \lambda} \Sigma^{\mu \nu} \Sigma^{\kappa \lambda}+\frac{\lambda}{2} F_{\mu \nu} \Sigma^{\mu \nu} .
$$

Using this nonminimal Hamiltonian in the brackets to construct equations of motion, we get

$$
\begin{aligned}
\pi_{\mu}= & m g_{\mu \nu} u^{\nu}, \\
m g_{\mu \nu} D_{\tau} u^{\nu}= & \frac{1}{2} \Sigma^{\kappa \lambda} R_{\kappa \lambda \mu \nu} u^{\nu}+q F_{\mu \nu} u^{\nu}-\frac{\kappa}{4} \Sigma^{\rho \sigma} \Sigma^{\kappa \lambda} \nabla_{\mu} R_{\rho \sigma \kappa \lambda} \\
& -\frac{\lambda}{2} \Sigma^{\kappa \lambda} \nabla_{\mu} F_{\kappa \lambda},
\end{aligned}
$$

and

$$
D_{\tau} \Sigma^{\mu \nu}=\left(\kappa \Sigma^{\rho \sigma} R_{\rho \sigma \lambda}^{\mu}+\lambda F_{\lambda}^{\mu}\right) \Sigma^{\nu \lambda}-\left(\kappa \Sigma^{\rho \sigma} R_{\rho \sigma \lambda}^{\nu}+\lambda F_{\lambda}^{\nu}\right) \Sigma^{\mu \lambda} .
$$

Remarkably, using Eqs. (15) and (16) and the Bianchi identities for $F_{\mu \nu}$ and $R_{\mu \nu \kappa \lambda}$, it is straightforward to generalize the theorem of Ref. [11], that any constant of motion (13) remains a constant of motion in the presence of Stern-Gerlach forces,

$$
\begin{aligned}
\left\{J, H_{\mathrm{SG}}\right\}= & \kappa \Sigma^{\mu \nu} \Sigma^{\rho \sigma}\left(-\frac{1}{4} \alpha^{\lambda} \nabla_{\lambda} R_{\rho \sigma \mu \nu}+R_{\rho \sigma \mu}{ }^{\lambda} \beta_{\lambda \nu}\right) \\
& +\lambda \Sigma^{\mu \nu}\left(-\frac{1}{2} \alpha^{\lambda} \nabla_{\lambda} F_{\mu \nu}+F_{\mu}{ }^{\lambda} \beta_{\lambda \nu}\right)=0 .
\end{aligned}
$$

In particular, in Schwarzschild and Reissner-Nordstrøm backgrounds the energy $E$, Eq. (19), and all three components of the full total angular momentum J, Eqs. (20), remain constants of motion for spinning neutral and charged particles.

The existence of these constants of motion enable us to perform a similar analysis of orbits as in the case of the minimal Hamiltonian. As an example we solve for plane circular orbits in a Schwarzschild background similar to those discussed in Sec. V for the minimal case. Again we take the plane of the orbit to be the equatorial plane $\theta=\pi / 2$ with $u^{\theta}=u^{r}=0$ and $\Sigma^{\theta \nu}=0$ for all values of $\nu$. Then the conservation laws reduce to the same form as in Eqs. (19) and (21),

$$
E=m\left(1-\frac{2 M}{R}\right) u^{t}-\frac{M}{R^{2}} \Sigma^{t r}, \quad J=m R^{2} u^{\varphi}+R \Sigma^{r \varphi}
$$

The Hamiltonian constraint now reads

$$
H=H_{0}+H_{\mathrm{SG}}=-\frac{m}{2},
$$

which becomes

$$
\begin{aligned}
& -\left(1-\frac{2 M}{R}\right) u^{t 2}+R^{2} u^{\varphi 2}+1 \\
& =-\frac{2 \kappa M}{m}\left[\frac{2}{R^{3}} \Sigma^{t r 2}+\frac{\Sigma^{r \varphi 2}}{R-2 M}-\frac{1}{R}\left(1-\frac{2 M}{R}\right) \Sigma^{t \varphi 2}\right] .
\end{aligned}
$$

Next the total spin is

$$
I=-\Sigma^{t r 2}-R^{2}\left(1-\frac{2 M}{R}\right) \Sigma^{t \varphi 2}+\frac{R^{2} \Sigma^{r \varphi 2}}{1-\frac{2 M}{R}}
$$

a constant. These constraints plus the vanishing of the radial acceleration $\dot{u}^{r}=0$ imply that the angular velocity and the time-dilation factor are constant, in fact causing $\Sigma^{t \varphi}$ to vanish,

$$
\dot{u}^{t}=\dot{u}^{\varphi}=\Sigma^{t \varphi}=0 .
$$

Then one finds a quartic equation for the angular velocity in terms of the radius $R$ and the angular momentum $J=m \eta$, which generalizes Eq. (37),

$$
\begin{aligned}
& \left(1-\frac{2 M}{R}\right)^{2} R u^{\varphi}\left[\frac{2 M^{2} \eta}{R^{3}}-M u^{\varphi}+M \eta u^{\varphi 2}+\left(R^{2}-6 M R+6 M^{2}\right) R u^{\varphi 3}\right] \\
& =\kappa m A+\kappa^{2} m^{2} B+\kappa^{3} m^{3} C
\end{aligned}
$$


where the coefficients $(A, B, C)$ are quartic polynomials in $u^{\varphi}$ themselves; the explicit expressions are given in Appendix C. After solving this equation for $u^{\varphi}$, the energy $E=m \varepsilon$ and the values of $u^{t}, \Sigma^{t r}$, and $\Sigma^{r \varphi}$ can also be determined.

\section{ENERGY-MOMENTUM TENSOR}

In the previous sections the equations of motion for a relativistic spinning particle were obtained starting from a closed set of brackets (5) and the choice of a Hamiltonian. The same equations can be derived by energy-momentum conservation using an appropriate energy-momentum tensor $[36,37]$. This tensor then also defines the source term in the Einstein equations to compute the backreaction of the particle on the spacetime geometry; indeed, the Einstein equations require the energy-momentum tensor to be divergence-free

$$
\begin{aligned}
G_{\mu \nu} & =R_{\mu \nu}-\frac{1}{2} g_{\mu \nu} R=-8 \pi G T_{\mu \nu} \Rightarrow \nabla^{\mu} G_{\mu \nu} \\
& =-8 \pi \nabla^{\mu} T_{\mu \nu}=0 .
\end{aligned}
$$

This identity is to be guaranteed by the equations of motion. For a neutral particle described by the minimal Hamiltonian, this follows by taking ${ }^{2}$

$$
\begin{aligned}
T_{0}^{\mu \nu}= & m \int d \tau u^{\mu} u^{\nu} \frac{1}{\sqrt{-g}} \delta^{4}(x-X) \\
& +\frac{1}{2} \nabla_{\lambda} \int d \tau\left(u^{\mu} \Sigma^{\nu \lambda}+u^{\nu} \Sigma^{\mu \lambda}\right) \frac{1}{\sqrt{-g}} \delta^{4}(x-X) .
\end{aligned}
$$

The covariant divergence of $T_{0}^{\mu \nu}$ is

$$
\begin{aligned}
\nabla_{\mu} T_{0}^{\mu \nu}= & \int d \tau\left(m \frac{D u^{\nu}}{D \tau}-\frac{1}{2} \Sigma^{\kappa \lambda} R_{\kappa \lambda}{ }_{\mu}{ }^{\mu} u^{\mu}\right) \frac{1}{\sqrt{-g}} \delta^{4}(x-X) \\
& +\frac{1}{2} \nabla_{\lambda} \int d \tau \frac{D \Sigma^{\nu \lambda}}{D \tau} \frac{1}{\sqrt{-g}} \delta^{4}(x-X)=0
\end{aligned}
$$

and vanishes upon applying the equations of motion (7) and (8) with $q=0$. Similarly, for a particle subject to the gravitational Stern-Gerlach force with the hamiltonian $H_{0}+H_{\mathrm{SG}}$ the correct expression is

$$
T^{\mu \nu}=T_{0}^{\mu \nu}+\kappa T_{1}^{\mu \nu},
$$

where

\footnotetext{
${ }^{2}$ We define the delta function as a scalar density of weight $1 / 2$, such that for scalar functions $f(x)$

$$
\int d^{4} y \delta^{4}(x-y) f(y)=f(x) .
$$
}

$$
\begin{aligned}
T_{1}^{\mu \nu}= & \frac{1}{2} \nabla_{\kappa} \nabla_{\lambda} \int d \tau\left(\Sigma^{\mu \lambda} \Sigma^{\kappa \nu}+\Sigma^{\nu \lambda} \Sigma^{\kappa \mu}\right) \frac{1}{\sqrt{-g}} \delta^{4}(x-X) \\
& +\frac{1}{4} \int d \tau \Sigma^{\rho \sigma}\left(R_{\rho \sigma \lambda}{ }^{\nu} \Sigma^{\lambda \mu}+R_{\rho \sigma \lambda}{ }^{\mu} \Sigma^{\lambda \nu}\right) \frac{1}{\sqrt{-g}} \delta^{4}(x-X) .
\end{aligned}
$$

Again performing standard operations from tensor calculus including Ricci and Bianchi identities leads to the result

$$
\begin{aligned}
\nabla_{\mu} T_{1}^{\mu \nu}= & \frac{1}{4} \int d \tau \nabla^{\nu} R_{\rho \sigma \kappa \lambda} \Sigma^{\rho \sigma} \Sigma^{\kappa \lambda} \frac{1}{\sqrt{-g}} \delta^{4}(x-X) \\
& +\frac{1}{2} \nabla_{\lambda} \int d \tau \Sigma^{\rho \sigma}\left(R_{\rho \sigma \kappa}{ }^{\lambda} \Sigma^{\kappa \nu}-R_{\rho \sigma \kappa}{ }^{\nu} \Sigma^{\kappa \lambda}\right) \\
& \times \frac{1}{\sqrt{-g}} \delta^{4}(x-X) .
\end{aligned}
$$

Combining this with the expression (69) for $\nabla_{\mu} T_{0}^{\mu \nu}$ it follows that the divergence of the full energy-momentum tensor vanishes

$$
\nabla_{\mu}\left(T_{0}^{\mu \nu}+\kappa T_{1}^{\mu \nu}\right)=0
$$

provided the nonminimal equations of motion (59) and (60) hold. Finally, one can also take into account the electromagnetic Lorentz and Stern-Gerlach forces by additional contributions

$$
\begin{aligned}
T_{\mu \nu}^{e m}= & F_{\mu}{ }^{\lambda} F_{\nu \lambda}-\frac{1}{4} g_{\mu \nu} F_{\kappa \lambda} F^{\kappa \lambda} \\
& -\frac{\lambda}{2} g_{\mu \nu} \int d \tau F_{\kappa \lambda} \Sigma^{\kappa \lambda} \frac{1}{\sqrt{-g}} \delta^{4}(x-X) .
\end{aligned}
$$

\section{DISCUSSION AND SUMMARY}

In this paper we have developed a covariant Hamiltonian framework for spinning particles in gravitational and electromagnetic background fields. One of its strong points is that it does not require an a priori choice of Hamiltonian; hence it can be applied to a large variety of models of relativistic spin dynamics. We have discussed in particular the case of the minimal kinetic Hamiltonian $H_{0}$ defined in Eq. (6) and its extension with a Stern-Gerlach type interaction Hamiltonian $H_{\mathrm{SG}}$ defined in Eq. (58). Other extensions are possible in principle. For example, one could introduce more covariant tensor fields allowing general expressions of the form

$$
H_{\text {eff }}=\frac{1}{2 m} g^{\mu \nu} \pi_{\mu} \pi_{\nu}+K_{\mu \nu}^{\lambda} \pi_{\lambda} \Sigma^{\mu \nu}+\frac{1}{2} M_{\mu \nu \kappa \lambda} \Sigma^{\mu \nu} \Sigma^{\kappa \lambda},
$$

where $K_{\mu \nu}^{\lambda}$ and $M_{\mu \nu \kappa \lambda}$ receive contributions from additional fields and/or geometric structures like torsion and 
curvature. Alternatively the equations of motion for spinning particles can be derived from the Einstein equations using appropriate energy-momentum tensors; we showed this explicitly for simple spinning particles with minimal or Stern-Gerlach dynamics.

We have applied our formalism to the case of spinning particles in Schwarzschild and Reissner-Nordstrøm backgrounds. We have found interesting new effects. For example, the periastron of spinning particles in bound orbits is not only subject to an angular shift, but the point of closest approach shows radial variations as well. Also the radius of the innermost stable circular orbit changes with spin; over a wide range of spin values $-0.5 M<$ $\sigma<0.5 M$ it is quite close to the orbit of minimal orbital angular momentum, but only for spinless particles do the two actually coincide. In addition we find an absolute minimal ISCO $R \simeq 4.3 M$ at an intermediate point between low and high spin values; beyond this the crossover to instability occurs on a different branch of stability limits, but these values are in the regime where the backreaction of the spinning particle on the spacetime geometry can no longer be neglected. For extreme mass ratio binaries with typical mass ratios $m / M<10^{-4}$ this limit is never reached, and the ISCO coincides to good approximation with the circular orbit of minimal orbital angular momentum.

With the formalism in hand there are still many open problems and applications to be studied. First of all, it would be interesting to consider the effect of spin on the emission of gravitational waves, as well as the backreaction of gravitational radiation on the spin dynamics. But also the generalization of our results to the case of nonminimal Hamiltonians and to Kerr backgrounds with the associated spin-spin coupling may have important astrophysical applications. Finally our approach is based on the idealization of compact spinning bodies as pointlike test particles. It may be refined to apply to finite-size bodies by including higher mass multipoles. All of this is left for future investigation.

\section{ACKNOWLEDGMENTS}

For G. d'A. and J.W. v. H. this work is part of the research programme Gravitational Physics of the Foundation for Fundamental Research of Matter (FOM) in the Netherlands.

\section{APPENDIX A: REISSNER-NORDSTRØM GEOMETRY}

In this appendix we collect the expressions for the components of the connection and Riemann curvature tensor used in the main body of the paper.

\section{Connection}

From the line element (17) one derives the following expressions for the connection coefficients:

$$
\begin{aligned}
\Gamma_{r t}{ }^{t} & =-\Gamma_{r r}{ }^{r}=\frac{M r-Q^{2}}{r\left(r^{2}-2 M r+Q^{2}\right)}, \\
\Gamma_{t t}{ }^{r} & =\frac{1}{r^{5}}\left(M r-Q^{2}\right)\left(r^{2}-2 M r+Q^{2}\right), \\
\Gamma_{\varphi \varphi}{ }^{r} & =\sin ^{2} \theta \Gamma_{\theta \theta}{ }^{r}=-\frac{\sin ^{2} \theta}{r}\left(r^{2}-2 M r+Q^{2}\right), \\
\Gamma_{r \theta} \theta & =\Gamma_{r \varphi}^{\varphi}=\frac{1}{r}, \\
\Gamma_{\theta \varphi}{ }^{\varphi} & =\frac{\cos \theta}{\sin \theta}, \quad \Gamma_{\varphi \varphi} \theta=-\sin \theta \cos \theta .
\end{aligned}
$$

\section{Curvature components}

The corresponding curvature two-form $R_{\mu \nu}=$ $\frac{1}{2} R_{\kappa \lambda \mu \nu} d x^{\kappa} \wedge d x^{\lambda}$ has the following components:

$$
\begin{aligned}
R_{t r} & =\frac{1}{r^{4}}\left(2 M r-3 Q^{2}\right) d t \wedge d r, \\
R_{t \theta} & =-\frac{1}{r^{4}}\left(M r-Q^{2}\right)\left(r^{2}-2 M r+Q^{2}\right) d t \wedge d \theta, \\
R_{t \varphi} & =-\frac{1}{r^{4}}\left(M r-Q^{2}\right)\left(r^{2}-2 M r+Q^{2}\right) \sin ^{2} \theta d t \wedge d \varphi, \\
R_{r \theta} & =\frac{M r-Q^{2}}{r^{2}-2 M r+Q^{2}} d r \wedge d \theta, \\
R_{r \varphi} & =\frac{M r-Q^{2}}{r^{2}-2 M r+Q^{2}} \sin ^{2} \theta d r \wedge d \varphi, \\
R_{\theta \varphi} & =-\left(2 M r-Q^{2}\right) \sin ^{2} \theta d \theta \wedge d \varphi .
\end{aligned}
$$

\section{APPENDIX B: COEFFICIENTS OF THE DEVIATION EQUATIONS}

The deviation equations for planar orbits of spinning particles in Reissner-Nordstrøm spacetime with respect to a reference circular orbit $r=R$ can be written in the form (41). Here we provide the explicit expressions for the coefficients:

$$
\begin{aligned}
& a=\frac{M R-Q^{2}}{R^{3}} u^{\varphi}, \\
& b=\frac{\left.\left(2 M R-3 Q^{2}\right)\left(R^{2}-2 M R+Q^{2}\right)\right)}{R^{3}\left(M R-Q^{2}\right)} u^{t}, \\
& c=-\frac{\left(M R-Q^{2}\right) m u^{t}}{R^{2}\left(R^{2}-2 M R+Q^{2}\right)}, \quad d=\frac{R^{2} m u^{\varphi}}{M R-Q^{2}},
\end{aligned}
$$

and furthermore 


$$
\begin{aligned}
\alpha= & \frac{\left(2 M R^{2}(R-M)-R Q^{2}(3 R-4 M)-Q^{4}\right)}{R\left(M R-Q^{2}\right)\left(R^{2}-2 M R+Q^{2}\right)} u^{t} \\
& -\frac{R\left(2 M R-3 Q^{2}\right)}{\left(M R-Q^{2}\right)\left(R^{2}-2 M R+Q^{2}\right)} \varepsilon+\frac{q Q}{m} \frac{M R-2 Q^{2}}{\left(M R-Q^{2}\right)\left(R^{2}-2 M R+Q^{2}\right)}, \\
\beta= & -\frac{\left(M R-Q^{2}\right)}{m R^{2}} u^{\varphi}, \\
\gamma= & \frac{2 R^{2}-5 M R+3 Q^{2}}{R\left(R^{2}-2 M R+Q^{2}\right)} u^{\varphi}+\frac{\left(M R-Q^{2}\right)}{R^{3}\left(R^{2}-2 M R+Q^{2}\right)} \eta, \\
\zeta= & -\frac{\left(R^{2}-2 M R+Q^{2}\right)\left(M R-Q^{2}\right)}{m R^{6}} u^{t}, \\
\kappa= & -\frac{\left(2 M R-3 Q^{2}\right)\left(R^{2}-2 M R+Q^{2}\right)}{R^{3}\left(M R-Q^{2}\right)} \varepsilon+\frac{q Q}{m} \frac{\left(M R-2 Q^{2}\right)\left(R^{2}-2 M R+Q^{2}\right)}{R^{4}\left(M R-Q^{2}\right)}, \\
\lambda= & \frac{2\left(M R^{2}-Q^{2}(2 R-M)\right)}{M R-Q^{2}} u^{\varphi}-\frac{M R-Q^{2}}{R^{3}} \eta,
\end{aligned}
$$

and also

$$
\begin{aligned}
\mu= & -\frac{2 M^{2} R^{3}(R-3 M)-3 M R^{2} Q^{2}(2 R-7 M)+R Q^{4}(3 R-20 M)+6 Q^{6}}{R^{4}\left(M R-Q^{2}\right)^{2}} \\
& +\frac{2 M^{2} R^{3}(R-4 M)-2 M R^{2} Q^{2}(3 R-14 M)+R Q^{4}(3 R-28 M)+9 Q^{6}}{R^{4}\left(M R-Q^{2}\right)^{2}} \varepsilon u^{t} \\
& -\frac{q Q}{m} \frac{2 M^{2} R^{3}(R-3 M)-M R^{2} Q^{2}(7 R-24 M)+R Q^{4}(4 R-25 M)+8 Q^{6}}{R^{5}\left(M R-Q^{2}\right)^{2}} u^{t} \\
& +\frac{M^{2}\left(R^{2}-Q^{2}\right)-2 M R Q^{2}+2 Q^{4}}{\left(M R-Q^{2}\right)^{2}} u^{\varphi 2}+\frac{2 M R-3 Q^{2}}{R^{4}} \eta u^{\varphi}, \\
\nu= & \frac{R^{2}(R-M)(R-3 M)+2 R Q^{2}(R-M)}{\left(M R-Q^{2}\right)\left(R^{2}-2 M R+Q^{2}\right)} m u^{\varphi}+\frac{M R-Q^{2}}{R^{2}\left(R^{2}-2 M R+Q^{2}\right)} m \eta, \\
\sigma= & \frac{R(R-M)\left(R^{2}-3 M R+2 Q^{2}\right)}{\left(M R-Q^{2}\right)\left(R^{2}-2 M R+Q^{2}\right)} m u^{t}-\frac{R^{2}}{M R-Q^{2}} m \varepsilon+\frac{q Q R}{M R-Q^{2}}, \\
\chi= & \frac{\left(M R^{2}-Q^{2}(2 R-M)\right)\left(R^{2}\left(R^{2}-4 M R+5 M^{2}\right)+2 Q^{2}\left(R^{2}-3 M R\right)+2 Q^{4}\right)}{\left(M R-Q^{2}\right)^{2}\left(R^{2}-2 M R+Q^{2}\right)^{2}} m u^{\varphi} u^{t} \\
& -\frac{M R^{2}(3 R-4 M)-Q^{2} R(4 R-7 M)-2 Q^{4}}{R^{3}\left(R^{2}-2 M R+Q^{2}\right)^{2}} \\
& -\frac{R\left(M R-2 Q^{2}\right)}{\left(M R-Q^{2}\right)^{2}} m \varepsilon u^{\varphi}-\frac{q Q^{3} u^{\varphi}}{\left(M R-Q^{2}\right)^{2}} .
\end{aligned}
$$

Of course, the corresponding expressions for Schwarzschild spacetime are obtained automatically by taking $Q=0$.

\section{APPENDIX C: CIRCULAR ORBITS WITH NONMINIMAL HAMILTONIAN}

In Sec. VIII we derived an equation for circular orbits for test particles in the presence of Stern-Gerlach interactions, Eq. (66). In this appendix we give the explicit expressions for the quantities $A, B$, and $C$, which are polynomials in the angular velocity $u^{\varphi}$ for given values of radius $R$ and angular momentum per unit of mass $\eta$. They read as follows: 


$$
\begin{aligned}
A= & \frac{M\left(\eta-R^{2} u^{\varphi}\right)}{R^{2}}\left\{\frac{6 M^{2} \eta}{R^{3}}\left(1-\frac{2 M}{R}\right)\left(2-\frac{m}{M}\right)\right. \\
& -4 M u^{\varphi}\left[3\left(1-\frac{2 M}{R}\right)\left(1-\frac{M}{R}-\frac{m}{2 R}\right)+\frac{M \eta^{2}}{R^{3}}\left(1-\frac{3 M}{R}\right)\right] \\
& +\eta R u^{\varphi 2}\left[\frac{2 M}{R}\left(7-\frac{16 M}{R}\right)-\frac{3 m}{M}\left(1-\frac{2 M}{R}\right)\left(1-\frac{4 M}{R}+\frac{6 M^{2}}{R^{2}}\right)\right] \\
& \left.+12 R^{3} u^{\varphi 3}\left[1-\frac{49 M}{6 R}+\frac{19 M^{2}}{R^{2}}-\frac{13 M^{3}}{R^{3}}+\frac{m}{4 M}\left(1-\frac{6 M}{R}+\frac{14 M^{2}}{R^{2}}-\frac{12 M^{3}}{R^{3}}\right)\right]\right\}, \\
B= & \frac{12 M\left(\eta-R^{2} u^{\varphi}\right)^{2}}{R^{3}}\left\{\frac{3 M^{2}}{R^{2}}\left(1-\frac{2 M}{R}\right)+\frac{2 M^{3} \eta^{2}}{R^{5}}-\frac{M \eta}{R} u^{\varphi}\left[\frac{M}{R}\left(5-\frac{6 M}{R}\right)-\frac{3 m}{M}\left(1-\frac{2 M}{R}\right)^{2}\right]\right. \\
& \left.-M R u^{\varphi 2}\left[3-\frac{20 M}{R}+\frac{26 M^{2}}{R^{2}}+\frac{3 m}{M}\left(1-\frac{2 M}{R}\right)^{2}\right]\right\}, \\
C= & \frac{72 M^{3}\left(\eta-R^{2} u^{\varphi}\right)^{4}}{R^{7}}\left[\frac{M}{R}-\frac{3 m}{2 M}\left(1-\frac{2 M}{R}\right)\right] .
\end{aligned}
$$

[1] W. de Sitter, On Einstein's theory of gravitation and its astronomical consequences, Mon. Not. R. Astron. Soc. 76, 699 (1916).

[2] L. Thomas, The motion of a spinning electron, Nature (London) 117, 514 (1926).

[3] J. Frenkel, Die Elektrodynamik des rotierenden Elektrons, Z. Phys. 37, 243 (1926).

[4] M. Mathisson, Neue mechanik materieller systeme, Acta Phys. Pol. 6, 163 (1937).

[5] A. Papapetrou, Spinning test particles in general relativity. 1., Proc. R. Soc. A 209, 248 (1951).

[6] V. Fock and D. Ivanenko, Über eine mögliche geometrische Deutung der relativistischen Quantentheorie, Z. Phys. 54, 798 (1929).

[7] W. Dixon, Dynamics of extended bodies in general relativity. I. Momentum and angular momentum, Proc. R. Soc. A 314, 499 (1970).

[8] R. M. Wald, Gravitational spin interaction, Phys. Rev. D 6, 406 (1972).

[9] A. J. Hanson and T. Regge, The relativistic spherical top, Ann. Phys. (N.Y.) 87, 498 (1974).

[10] O. Semerak, Spinning test particles in a Kerr field. I., Mon. Not. R. Astron. Soc. 308, 863 (1999).

[11] G. d'Ambrosi, S. Satish Kumar, and J. W. van Holten, Covariant hamiltonian spin dynamics in curved space-time, Phys. Lett. B 743, 478 (2015).

[12] G. Schaefer, Gravitomagnetic effects, Gen. Relativ. Gravit. 36, 2223 (2004).

[13] K. Kyrian and O. Semerak, Spinning test particles in a Kerr field. II., Mon. Not. R. Astron. Soc. 382, 1922 (2007).

[14] R. Plyatsko, O. Stefanyshyn, and M. Fenyk, MathissonPapapetrou-Dixon equations in the Schwarzschild and Kerr backgrounds, Classical Quantum Gravity 28, 195025 (2011).

[15] J. Steinhoff, Canonical formulation of spin in general relativity, Ann. Phys. (Berlin) 523, 296 (2011).

[16] L. F. O. Costa, J. Natario, and M. Zilhao, Spacetime dynamics of spinning particles-exact gravito-electromagnetic analogies, arXiv:1207.0470v2.

[17] I. Khriplovich, Spinning relativistic particles in external fields, Acta Phys. Pol. B Proc. Suppl. 1, 197 (2008).

[18] I. Khriplovich and A. Pomeransky, Equations of motion of spinning relativistic particle in external fields, Surv. High Energy Phys. 14, 145 (1999).

[19] I. Khriplovich and A. Pomeransky, Equations of motion of spinning relativistic particle in external fields, J. Exp. Theor. Phys. 86, 839 (1998).

[20] I. Khriplovich and A. Pomeransky, Gravitational interaction of spinning bodies, center-of-mass coordinate and radiation of compact binary systems, Phys. Lett. A 216, 7 (1996).

[21] I. Khriplovich, Particle with internal angular momentum in a gravitational field, Sov. Phys. JETP 69, 217 (1989).

[22] F. Pirani, On the physical significance of the Riemann tensor, Acta Phys. Pol. 15, 389 (1956).

[23] F. Berezin and M. Marinov, Particle spin dynamics as the Grassmann variant of classical mechanics, Ann. Phys. (N.Y.) 104, 336 (1977).

[24] L. Brink, P. Di Vecchia, and P. S. Howe, A Lagrangian formulation of the classical and quantum dynamics of spinning particles, Nucl. Phys. B118, 76 (1977).

[25] J. W. van Holten and R. H. Rietdijk, Symmetries and motions in manifolds, J. Geom. Phys. 11, 559 (1993). 
[26] D. Bini, A. Geralico, and R. T. Jantzen, Spin-geodesic deviations in the Schwarzschild spacetime, Gen. Relativ. Gravit. 43, 959 (2011).

[27] G. Koekoek and J. W. van Holten, Epicycles and Poincaré resonances in general relativity, Phys. Rev. D 83, 064041 (2011).

[28] G. Koekoek and J. W. van Holten, Geodesic deviations: Modeling extreme mass-ratio systems and their gravitational waves, Classical Quantum Gravity 28, 225022 (2011).

[29] R. Kerner, J. W. van Holten, and R. Colistete, Jr., Relativistic epicycles: Another approach to geodesic deviations, Classical Quantum Gravity 18, 4725 (2001).

[30] S. Suzuki and K. Maeda, Innermost stable orbit of a spinning particle in Kerr spacetime, Phys. Rev. D 58, 023005 (1998).

[31] L. Blanchet and B. R. Iyer, Third post-Newtonian dynamics of compact binaries: Equations of motion in the center-ofmass frame, Classical Quantum Gravity 20, 755 (2003).
[32] E. Hackmann, C. Lämmerzahl, Y. Obukhov, D. Puetzfeld, and I. Schaffer, Motion of spinning test bodies in Kerr spacetime, Phys. Rev. D 90, 064035 (2014).

[33] A. Barducci, R. Casalbuoni, and L. Lusanna, Supersymmetries and the pseudoclassical relativistic electron, Nuovo Cimento Soc. Ital. Fis. 35A, 377 (1976).

[34] J. W. van Holten, Theory of a charged spinning particle in a gravitational and electro-magnetic field, in Proceedings of the Seminar on Mathematical Structures in Field Theory 1986-1987, CWI-syllabus Vol. 26, edited by E. A. de Kerf and H. G. J. Pijls (CWI, Amsterdam, 1990).

[35] J.W. van Holten, On the electrodynamics of spinning particles, Nucl. Phys. B356, 3 (1991).

[36] J. Steinhoff and D. Puetzfeld, Multipolar equations of motion for extended test bodies in general relativity, Phys. Rev. D 81, 044019 (2010).

[37] J. W. van Holten, Spinning bodies in general relativity, arXiv:1504.04290v2. 